\title{
"Not getting worse" a qualitative study of patients perceptions of treatment goals in patients with heart failure
}

I. H. Kraai, K. M. Vermeulen, H. L. Hillege and Tiny Jaarsma

The self-archived postprint version of this journal article is available at Linköping University Institutional Repository (DiVA):

http://urn.kb.se/resolve?urn=urn:nbn:se:liu:diva-145774

N.B.: When citing this work, cite the original publication.

Kraai, I. H., Vermeulen, K. M., Hillege, H. L., Jaarsma, T., (2018), "Not getting worse" a qualitative study of patients perceptions of treatment goals in patients with heart failure, Applied Nursing

Research, 39, 41-45. https://doi.org/10.1016/j.apnr.2017.10.010

Original publication available at:

https://doi.org/10.1016/j.apnr.2017.10.010

Copyright: WB Saunders

http://www.elsevier.com/ 


\section{Highlights}

- This qualitative study provides new insights into how patients with HF perceive their treatment goals.

- "Not getting worse" was perceived by patients as the most important goal of treatment

- Other treatment goals of the participants included decreasing symptoms, improving physical function, avoiding readmission, and being able to live a normal life.

- More research is necessary to develop endpoints that are consistent with the perception of patients, useful for clinical practice, and measurable. 


\section{"Not getting worse" a qualitative study of patients perceptions of treatment goals in patients with heart failure}

\section{Abstract}

Background: Knowledge of patient reported outcomes helps to provide personalized care on a patient level, optimize care on a population level, and identify the most appropriate patient reported outcomes for clinical trials. To be able to provide personalized care to patients with $\mathrm{HF}$, it is important to know which treatment goals patients with HF consider the most important. This is particularly useful for interventions aimed at improving symptoms, functional status, or health-related quality of life.

Aim: The aim of this study was (1) to explore which treatment goals patients with heart failure (HF) perceive as important for their treatment and (2) to identify which treatment goals take priority over other treatment goals.

Methods: The study had a qualitative design and included one focus group interview with 6 patients with HF. Data were analyzed using qualitative content analysis.

Results: The overall theme of this study was found to be 'Not getting worse'. This theme was composed of 4 categories that described how the patients perceived the goals of treatment: to decrease symptoms, to improve physical function, to prevent readmission, and to live a normal life. None of the participants mentioned improved survival or being cured of their HF as a treatment goal.

Conclusion: 'Not getting worse' was perceived as the treatment goal that matters to patients with HF and is in line with currently used endpoints for the evaluation of a treatment in a clinical trial. More research is necessary to develop endpoints that are consistent with the perception of patients, useful for clinical practice, and measurable.

Keywords: Cardiovascular, Focus group, Patient centered care, Qualitative, Heart Failure 


\section{Introduction}

There is a growing recognition of the importance of patient preferences throughout the disease trajectory. Recently, patient-reported outcomes have been recognized as powerful tools to inform patients, clinicians, and policy-makers about morbidity and patient suffering, especially in chronic diseases. Medically defined treatment outcomes, such as readmission and mortality, might not be considered as the important outcomes by patients with heart failure (HF) and while some patients value survival as most important (Stewart et al, 2010), others might consider other outcomes to be of greater importance (I. H. Kraai et al., 2013; I. H. Kraai, Vermeulen, Hillege, \& Jaarsma, 2016).

Knowledge about patient-reported outcomes can help to provide personalized care on a patient level, optimize care on a population level, and identify the most appropriate patient-reported outcomes for clinical trials. This is in particularly useful for interventions aimed at improving symptoms, functional status, or health-related quality of life (HR-QoL) (Rahimi, Malhotra, Banning, \& Jenkinson, 2010). Whereas from a patient's perspective the treatment of $\mathrm{HF}$ is aimed at the relief of symptoms and improvement of health-related quality of life, trials have been primarily oriented at endpoints that evaluate mortality and readmissions (McMurray et al., 2012).

To be able to provide personalized care to patients with HF, it is important to know which treatment goals patients with HF consider to be the most important. The aim of this study was (1) to explore which treatment goals patients with HF perceive as important for their treatment and (2) to identify which treatment goals take priority over other treatment goals.

\section{Methods}

The study had a qualitative design using a focus group interview with 6 patients with HF. Data were analyzed using qualitative content analysis.

\section{Participants and sampling}

The participating patients were recruited at a university hospital in the Netherlands between February and March 2014. Participants were invited based on their participation in a previous 
study on patient preferences (I. H. Kraai et al., 2013). In short, the inclusion criteria of the previous study were: diagnosis of HF, New York Heart Association functional (NYHA) classification I-IV, and age 50 years or older. Patients were excluded in case of impaired cognition, inability to speak the Dutch language, or not being able to understand informed consent. Demographics and medical history were assessed with a short questionnaire.

Twelve patients received a phone call from the researchers in which they were invited to participate. Six patients declined to participate because they felt participation would be too exhausting $(n=1)$; they were unavailable due to conflicting appointments $(n=3)$; they were unable to visit the location due to their physical condition $(n=1)$; or they were not interested in participating $(n=1)$. A total of 6 patients participated in the focus group session. The mean age of the participants was 70 (range 51-80) years (Table 1). The duration of HF ranged between 2-10 years, and the most common etiology of the HF was non-ischemic. All of the participants were diagnosed with one or more comorbidities. On the day of the focus group session, participant P1 suffered from dyspnea during rest and during exertion, experienced heart palpitations, tiredness and nocturnal dyspnea (assessed by self-completion questionnaire). Participants P5 and P6 also suffered from tiredness on the day of the focus group session; participant P5 and participant P4 experienced dyspnea during exertion. Two participants (P2 and P3) did not suffer from any of these symptoms on the day of the focus group session.

\section{Procedure}

The focus group session took place in a comfortable and quiet conversation room in the hospital (Simpson, Farris, Johnson, \& Tsuyuki, 2000). The focus group session lasted approximately 2.5 hours with a coffee break included. The session was moderated by a researcher with a nursing background (XXX). She directed the discussion and facilitated the conversation flow. Another researcher (XXX) with a background in quality of life studies attended the interviews as an observer, wrote important statements on a flip chart, and occasionally asked additional questions in order to gain a deeper understanding of the topics discussed. The flip chart provided an overview of the topics raised to the participants during the session.

Before the session started, the participants gave their written informed consent for the participation in the study. The session started with a short introduction to make the 
participants feel comfortable and to introduce the participants to each other. An interview guide was used during the session to make sure that all topics were discussed. The main topic was the most relevant treatment outcome according to the participating patients with HF. They were asked to reflect on the treatment goal that was currently the most important for them as well as the treatment goals that might be important in the near future and in other (hypothetical) situations (Table 2).

Open-ended questions were used during the session and sometimes follow-up questions were formulated, such as "What is your view on this treatment goal?"; "Do you have anything to add?" and "What is your opinion about this?" At the end of the session, the moderator summarized the points that were discussed and asked questions, such as "Would you like to add something that was not discussed?" and "Have we summarized this correctly?" The observer made field notes during the summary. The session was audio-recorded with permission of the participants The field notes could be consulted during the analysis in case the recordings were not clear. The audio-recording started after the introduction to make sure the names of the participants were not recorded. Afterwards, the session was transcribed verbatim by XXX. Coughing and remarks not relevant to the discussion were not transcribed. The transcription was read and checked for authentication by XXX. Our study conforms to the principles outlined in the Declaration of Helsinki. The Medical Ethics Committee provided a waiver for the study.

\section{Analysis}

The transcription of the session was read several times by both researchers to be able to perform the qualitative content analysis. The qualitative content analysis, as described by Graneheim et al. 2004 (Graneheim \& Lundman, 2004), uses the categories meaning unit, condensation, code, category, and theme. These categories were employed to analyze the transcription on its latent meaning. First, we defined the meaning units in the transcription. Meaning units are words, sentences, or paragraphs with aspects related to each other through their content and context. Second, we shortened the meaning units while preserving the core to have the condensation of the transcription. Third, we abstracted the condensed text to codes, which is also the label of the meaning unit. Fourth, subcategories and categories were created, which are seen as the core features of qualitative content analysis. The fifth and last step consisted of identifying the themes. A theme is considered as a thread of an 
underlying meaning through, condensed meaning units, codes or categories on an interpretative level. An example of the content analysis can be found in Table 3. XXX and XXX analyzed the transcription separately and selected those meaning units from the transcription that were related to the aim of this study. Subsequently, both researchers discussed the items that did not correspond to the aim. All meaning units, condensations, codes, categories, and themes were discussed with a third researcher, a HF nurse researcher with experience in qualitative research $(X X X)$ and updated until consensus was reached.

\section{Trustworthiness}

Because a discussion during a focus group session always involves multiple opinions and entails a certain degree of interpretation, the content analysis was performed by two researchers and one independent researcher. During the content analysis, the written transcriptions were consulted on a regular basis and coding and categorization were discussed with the third researcher (XXX), who did not participate in the focus group session but was involved in designing the study and analyzing, interpreting, and drafting the article.

\section{Findings}

\section{Not getting worse}

Not getting worse was found to be the main theme of this study. The participants described that having HF meant they had to cope with numerous limitations and often had to make sacrifices, but that appropriate treatment could make them stay longer in a stable phase.

When debating the merits of a miracle pill, participants hoped such a pill would keep their condition stable for a longer period of time.

"Such a pill would be very nice indeed, if my condition were to remain the same." (P2)

"That my condition will not worsen further." (P1)

"To live independently for as long as possible, just stay calm." (P1)

"Just stay calm and keep on going." (P4)

The main theme of this study was composed of four categories that described how the participants perceived the goal of treatment: to decrease symptoms, to improve physical function, to prevent readmission, and to live a normal life (Figure 1). None of the participants 
mentioned improved survival or being cured of their HF as a treatment goal. Furthermore, we also asked the participants to assign a weight to the different categories. Participants in our focus group were not able to do this because they would like to see their life as a complete picture.

\section{To decrease symptoms}

The participants described that they suffered from symptoms associated with HF and wished for adequate treatment of their dyspnea and fatigue.

"I told my cardiologist that if I would stop feeling short of breath, I would hug him. He might not like that, but I would be very grateful" (P1)

"Is that really a goal of treatment for you, to have less dyspnea?" (Interviewer I)

"Yes, that really matters to me, and also dizziness: I want to feel less short of breath and less dizzy."(P1)

Symptoms impaired participants' daily functioning; they had, for example, trouble getting dressed and undressed and found it difficult to go up the stairs. These symptoms also made them feel insecure.

"I have been in a cardiac rehabilitation program twice to improve my physical condition and that went well. But then, at a certain moment, the cardiac rehabilitation program ends. And because I feel short of breath so often, I am afraid to do certain things. I also feel very dizzy and wobbly, which makes me feel insecure" (P1)

In addition to the physical symptoms, some participants described feelings of anxiety, disappointment, and depressive feelings related to their disease. Participants also experienced the burden of side effects of medication and worried about the amount of medication they have to take.

\section{To improve physical function}

Participants reported that they considered improving their physical function to be an important goal of their treatment. Some of the participants had participated in a rehabilitation program in the past and reported that such a program had really helped them. The program taught them among other things that retaining good physical function is 
imperative to remain independent. Participants, however, also noticed that their physical function declines over time, which worried them. They could not bike as far as they were used to, or could no longer walk up as many flights of stairs.

"I still try to ride my bicycle. Once I got on my bicycle on a windy day. I do not know whether you know the city road, but I was cycling there. I thought, is it me or is it the bicycle? I was cycling at a really slow pace. But yes, that is the condition, your physical capacity is diminished and you have to go up against the wind."(P4)

"You are out of shape; you need to build up your stamina."(P4)

"Or adapt."(P6)

"Yes, my bicycle has not changed. I recently said to my husband: I am not getting back on my bicycle. I am not making any progress; my bicycle is just so heavy. I blame the bicycle.

But it is not the bicycle's fault." (P3)

The participants expected that adequate treatment could help them maintain or maybe even improve their physical function. They expressed that one aspect of treatment was an exercise program offered by a rehabilitation center, a local sports center, or a physiotherapist. They were worried about the future because of increased healthcare costs and reduced reimbursements. Some participants felt that an important goal of their treatment should be to improve their physical function, enabling them to walk longer distances, for example. Others preferred a focus on stabilizing their current condition.

\section{To prevent readmission}

Participants reported that being admitted to the hospital was a very stressful experience. Although participants in general were content with the treatment and care provided during an acute admission, the hospitalization itself but the means of transportation to the hospital - via ambulance or sometimes helicopter - caused them a lot of stress.

"I just cannot stand hearing the siren of an ambulance, it is awful."(P3)

"It is awful. Whenever I see such a newly designed ambulance I remember that I was in there once, and I still have those images in my head. But the sound, I cannot stand that sound, I think it is awful."(P3)

After being admitted, participants were left to the care of others and were deeply worried about themselves and their family members. This had a profound impact on the participants. 
Consequently, they wanted their treatment to prevent this from ever happening ever again. Participants also reported that it was important for them to be able to live independently and make their own choices.

"The announcement of the physician that a readmission is not necessary is very important for me. Six months ago, a physician enthusiastically proposed an admission to the hospital followed by surgery so that I might live longer. But I would like to be treated with drugs and recover gradually, as befits my age." (P2)

After discharge, participants sometimes felt they were not ready to go home, and they also felt at loss with the changes in treatment as well as with their altered physical condition.

"As soon as you are admitted you want to crawl back home, but when you are discharged you want to come crawling back." (P1)

"And then you have to take care of all the medication yourself, and they give you all sorts of boxes. And when you fill a box, you keep wondering whether you are doing it right. And then you start thinking: this pill looks different from the one I took in the hospital." (P3)

\section{To live a normal life}

Participants expressed the wish to be included in life. Treatment should help them stay independent and live as normally as possible. Although the participants realized they could no longer perform all the activities they used to perform before being diagnosed with HF, they did express the wish to participate in work and social life. Some succeeded in this by adapting their work where necessary or by using assistive equipment (e.g., electric bicycle, rollator), which helped them to live as normally as possible.

"It is like I said before, the feeling of disappointment in yourself because this is it, and it is not going to change."(P6)

"And you really are confronted with yourself." (P1)

"And it is such a shame that you cannot do it anymore." (P5)

Participants also mentioned that healthcare providers need to stress the importance of lifestyle changes and adherence to therapy, especially in the first stage of the HF trajectory. Medication was seen as something that could not be avoided and could help in case of increasing symptoms of HF. They expressed that being able to take care of themselves and being involved in daily life was important, but that their HF makes this difficult. Participants 
recognized that treatment decreases their suffering and helps them live their life as normally as possible within the limits of their HF. HF and comorbidities (e.g., breast cancer, diabetes) made them vulnerable to losing their independence.

"And my 21\% heart function, which is rapidly decreasing."(P4)

"Yes that is getting less, isn't it." (P6)

"Well, as long as it does not decrease even further." (P1)

"Yes, but when you compare yourself with healthy people." (P4)

"Yes, but that is something that we cannot do either." (P6)

"Of course you cannot compare us with normal people." (P6)

\section{Discussion}

This qualitative study provides new insights into how patients with HF perceive their treatment goals. Not getting worse was found to be the main theme of this study. The treatment goals of the participants included decreasing symptoms, improving physical function, avoiding readmission, and being able to live a normal life.

The focus group session demonstrated that what patients consider important treatment goals do not always correspond to standard endpoints for the evaluation of a treatment in a clinical trial. In most of these trials, hospitalization and death are seen as primary outcomes, and health-related quality of life is only a secondary endpoint (McMurray et al., 2012; Packer, 2001; Rahimi et al., 2010). Participants in this focus group did not mention improving survival as a treatment goal, but they did mention the importance of preventing a hospital readmission. This was experienced by the participants as being related to 'not getting worse' and the fact that the participants experienced an acute readmission as very distressing. The chaotic medical environment of an acute readmission is known to influence the psychological well-being of patients dramatically (Edmondson, Shimbo, Ye, Wyer, \& Davidson, 2013). Moreover, after an acute readmission, patients not only need to recover from their acute illness, but they are also vulnerable to a wide range of adverse health events and suffer from stress from hospitalization, which places them at risk of being readmitted within 30 days (Edmondson, Green, Ye, Halazun, \& Davidson, 2014; Krumholz, 2013). Another 
adverse outcome of readmission might that patients become deconditioned, which affects their ability to comply with post-discharge instructions (Krumholz, 2013).

'Not getting worse' was reported as an important treatment goal by the participants in the focus group. 'Not getting worse' i.e. to prevent a patient with a progressive disease from worsening is challenging for healthcare providers. Some trial endpoints were identified by the participants as being important, while others, for instance, the wish to live a normal life, are not always evaluated in clinical trials. The ESC, AHA, and FDA recommend including patient- reported outcomes in the evaluation of interventions (Anker et al., 2014; Rumsfeld et al., 2013; US department of Health and Human Services Food and Drug Administration, 2009; Zannad et al., 2013). In a recent statement the ESC also recommended the use of a composite endpoint that includes death, hospitalization and another component, for example, HR-QoL (Zannad et al., 2013). A recent study, however, has found substantial differences between the preferences of patients and investigators regarding the weight of individual components in a composite endpoint (Stolker et al., 2014). Our study confirms this discrepancy because the participants mentioned the endpoint prevention of readmission in relation not getting worse. Although none of the participants mentioned that the goal of treatment should be improved survival, we do not want to implicate that this is not important to patients (Stewart, 2010). The clinical composite score proposed by Packer includes the term 'not getting worse,' which was expressed by participants in our focus group. The clinical composite score classifies each patient as 'improved,', 'unchanged,' or 'worse.' (Packer, 2001). The treatment goal of the participants in our focus group was met when the majority of patients scored 'improved' and 'unchanged'. A 'worse' score meant the treatment goal of the patients of 'not getting worse' was not met.

Participants in our focus group were not able to assign a weight to the different categories because they would like to see their life as a complete picture. These findings are consistent with another study in which patients with multimorbidity preferred a holistic approach to their treatment (Eckerblad, Theander, Ekdahl, Jaarsma, \& Hellstrom, 2015). More research is needed to develop endpoints that are measurable as well as consistent with patients' perceptions.

The concept of HR-QoL was not mentioned by the participants in the focus group. However, components that are included in general and disease-specific HR-QoL, such as 'decrease in symptoms,' 'improve physical function,' and 'to live a normal life,' were seen as 
important treatment goals by the participants. Further studies are needed to optimize the selection of patient- reported outcomes. In addition, this study showed that patients with HF wish to remain in a stable condition. It might be interesting to explore which interventions can provide stability. Telemonitoring, for example, can be used to monitor the condition of a patient at home safely and can reduce HF-related visits to the HF-outpatient clinic (I. Kraai et al., 2016). Moreover, telemonitoring offers the possibility to treat progression of the disease before the symptoms increase (de Vries et al., 2011).

This study has several limitations. First, the focus group session took place only once. Multiple sessions might lead to other results or confirm the results. Second, another approach to data collection (e.g., individual interviews) might have yielded more in-depth results. Third, the sample size was small which limits the generalizability of the results. However the data was rich, there were a lot of rich discussions and reflections during the focus group and all 6 patients contributed to the final results. Despite these limitations a strength of the study is the design. To our knowledge, this is the first study to use a focus group to explore which goals patients with HF perceive as important for their treatment. Furthermore, the design included a focus group session because we believe that group interaction has a positive influence on the discussion and more items will be discussed in depth. This is only possible when there is a pleasant atmosphere in the group, allowing the participants to talk freely. During this particular session the atmosphere was good and the participants felt comfortable sharing their views.

\section{Conclusion}

This study provides new insights into the perceptions of patients with HF regarding their treatment goals. 'Not getting worse' was perceived as the treatment goal that matters to patients with HF. Although frequently used endpoints in clinical trials, such as decrease in symptoms and improvement in physical function, were recognized by the participants as important, those endpoints are not always included in the designs of trials. More research is necessary to develop endpoints that are consistent with the perception of patients, useful for clinical practice, and measurable. 


\section{$\underline{\text { References }}$}

Anker, S. D., Agewall, S., Borggrefe, M., Calvert, M., Jaime Caro, J., Cowie, M. R., Kirchhof, P. (2014). The importance of patient-reported outcomes: A call for their comprehensive integration in cardiovascular clinical trials. European Heart Journal, 35(30), 2001-2009. doi:10.1093/eurheartj/ehu205 [doi]

de Vries, A. E., van der Wal, M. H., Bedijn, W., de Jong, R. M., van Dijk, R. B., Hillege, H. L., \& Jaarsma, T. (2011). Follow-up and treatment of an instable patient with heart failure using telemonitoring and a computerised disease management system: A case report. European Journal of Cardiovascular Nursing : Journal of the Working Group on Cardiovascular Nursing of the European Society of Cardiology, doi:10.1016/j.ejcnurse.2011.04.001

Eckerblad, J., Theander, K., Ekdahl, A., Jaarsma, T., \& Hellstrom, I. (2015). To adjust and endure: A qualitative study of symptom burden in older people with multimorbidity. Applied Nursing Research : ANR, 28(4), 322-327. doi:10.1016/j.apnr.2015.03.008 [doi]

Edmondson, D., Green, P., Ye, S., Halazun, H. J., \& Davidson, K. W. (2014). Psychological stress and 30-day all-cause hospital readmission in acute coronary syndrome patients: An observational cohort study. PloS One, 9(3), e91477. doi:10.1371/journal.pone.0091477 [doi]

Edmondson, D., Shimbo, D., Ye, S., Wyer, P., \& Davidson, K. W. (2013). The association of emergency department crowding during treatment for acute coronary syndrome with subsequent posttraumatic stress disorder symptoms. JAMA Internal Medicine, 173(6), 472474. doi:10.1001/jamainternmed.2013.2536 [doi] 
Graneheim, U. H., \& Lundman, B. (2004). Qualitative content analysis in nursing research: Concepts, procedures and measures to achieve trustworthiness. Nurse Education Today, 24(2), 105-112. doi:10.1016/j.nedt.2003.10.001 [doi]

Kraai, I., de Vries, A., Vermeulen, K., van Deursen, V., van der Wal, M., de Jong, R., . . Lesman, I. (2016). The value of telemonitoring and ICT-guided disease management in heart failure: Results from the IN TOUCH study. International Journal of Medical Informatics, 85(1), 53-60. doi:10.1016/j.ijmedinf.2015.10.001 [doi]

Kraai, I. H., Vermeulen, K. M., Hillege, H. L., \& Jaarsma, T. (2016). Perception of impairments by patients with heart failure. European Journal of Cardiovascular Nursing : Journal of the Working Group on Cardiovascular Nursing of the European Society of Cardiology, 15(2), 178185. doi:1474515115621194 [doi]

Kraai, I. H., Vermeulen, K. M., Luttik, M. L., Hoekstra, T., Jaarsma, T., \& Hillege, H. L. (2013). Preferences of heart failure patients in daily clinical practice: Quality of life or longevity? European Journal of Heart Failure, 15(10), 113-1121. doi:10.1093/eurjhf/hft071

Krumholz, H. M. (2013). Post-hospital syndrome--an acquired, transient condition of generalized risk. The New England Journal of Medicine, 368(2), 100-102. doi:10.1056/NEJMp1212324 [doi]

McMurray, J. J., Adamopoulos, S., Anker, S. D., Auricchio, A., Bohm, M., Dickstein, K., ... ESC Committee for Practice Guidelines. (2012). ESC guidelines for the diagnosis and treatment of acute and chronic heart failure 2012: The task force for the diagnosis and treatment of acute and chronic heart failure 2012 of the european society of cardiology. developed in 
collaboration with the heart failure association (HFA) of the ESC. European Journal of Heart Failure, 14(8), 803-869. doi:10.1093/eurjhf/hfs105; 10.1093/eurjhf/hfs105

Packer, M. (2001). Proposal for a new clinical end point to evaluate the efficacy of drugs and devices in the treatment of chronic heart failure. Journal of Cardiac Failure, 7(2), 176-182. doi:10.1054/jcaf.2001.25652

Rahimi, K., Malhotra, A., Banning, A. P., \& Jenkinson, C. (2010). Outcome selection and role of patient reported outcomes in contemporary cardiovascular trials: Systematic review. BMJ (Clinical Research Ed.), 341, c5707. doi:10.1136/bmj.c5707

Rumsfeld, J. S., Alexander, K. P., Goff, D. C.,Jr, Graham, M. M., Ho, P. M., Masoudi, F. A., . . . American Heart Association Council on Quality of Care and Outcomes Research, Council on Cardiovascular and Stroke Nursing, Council on Epidemiology and Prevention, Council on Peripheral Vascular Disease, and Stroke Council. (2013). Cardiovascular health: The importance of measuring patient-reported health status: A scientific statement from the american heart association. Circulation, 127(22), 2233-2249. doi:10.1161/CIR.0b013e3182949a2e; 10.1161/CIR.0b013e3182949a2e

Simpson, S. H., Farris, K. B., Johnson, J. A., \& Tsuyuki, R. T. (2000). Using focus groups to identify barriers to drug use in patients with congestive heart failure. Pharmacotherapy, 20(7), 823-829.

Stolker, J. M., Spertus, J. A., Cohen, D. J., Jones, P. G., Jain, K. K., Bamberger, E., . . Chan, P. S. (2014). Rethinking composite end points in clinical trials: Insights from patients and trialists. Circulation, 130(15), 1254-1261. doi:10.1161/CIRCULATIONAHA.113.006588 [doi] 
US department of Health and Human Services Food and Drug Administration. (2009). Guidance for industry: Patient-reported outcome measures, use in medical product development to support labelling claims. Retrieved from http://www.fda.gov/downloads/Drugs/GuidanceComplianceRegulatoryInformation/Guidan ces/UCM193282.pdf

Zannad, F., Garcia, A. A., Anker, S. D., Armstrong, P. W., Calvo, G., Cleland, J. G., . . McMurray, J. J. (2013). Clinical outcome endpoints in heart failure trials: A european society of cardiology heart failure association consensus document. European Journal of Heart Failure, 15(10), 1082-1094. doi:10.1093/eurjhf/hft095 [doi]

toevoegen: J Card Fail. 2010 Feb;16(2):106-13. Patient expectations from implantable defibrillators to prevent death in heart failure. Stewart GC1, Weintraub JR, Pratibhu PP, Semigran MJ, Camuso JM, Brooks K, Tsang SW, Anello MS, Nguyen VT, Lewis EF, Nohria A, Desai AS, Givertz MM, Stevenson LW.

Acknowlegdement: We would like to thank dr. M. Liljeroos for the critical comments related to the qualitative analysis

Conflicts of interest: none

Funding: This work was supported by the Dutch Ministry of Health, Welfare, and Sport. 
